\title{
Implantação de um software para controle da central de esterilização do curso de Odontologia da Universidade Positivo
}

\author{
Sabrina Rodrigues Silva*; Keli Gilliet*; Paula Porto Spada**; Tatiana Miranda Deliberador***; \\ Denise Piotto Leonardi***; Flares Baratto-Filho***; Aline Monise Sebastiani***; Rafaela Scariot*** \\ * Estudante de Graduação em Odontologia, Universidade \\ Positivo \\ ** Doutorando, Programa de Pós-graduação em Odontologia, \\ Universidade Positivo \\ *** Professor, Programa de Pós-graduação em Odontologia, \\ Universidade Positivo
}

Recebido em 11/10/2017. Aprovado em 08/07/2018.

\begin{abstract}
RESUMO
O objetivo deste trabalho foi descrever um método de controle de esterilização dos materiais utilizados pelos acadêmicos de Odontologia, bem como comparar a sua eficiência frente ao antigo método de controle. Um software foi criado pela Gestão de Tecnologia da Informação da Universidade Positivo, a partir de uma demanda interna do curso, a fim de aprimorar o controle da central de esterilização. O novo método permite que etiquetas com código de barras sejam colocadas externamente em todas as caixas dos alunos, controlando todo o fluxo de entrada e saída de materiais, gerando maior segurança e agilidade do processo de esterilização. Além disso, o sistema permite que o professor tenha acesso à lista de todo o material esterilizado pelo aluno, bem como a data, horário e número da autoclave na qual o material foi esterilizado. Para avaliar a satisfação com o método foram entregues questionários para os estudantes do último ano do curso de Odontologia, contendo perguntas subjetivas sobre o novo e sobre o antigo método de controle. De acordo com o questionário aplicado, demonstrou-se que novo método utilizado diminui os atrasos para atendimento e o número de materiais danificados e perdidos, aumenta a segurança e acelera o tempo para a colocação e retirada dos materiais $(\mathrm{p}<0,05)$. Na avalição geral, o método também se mostrou melhor quando comparado ao antigo $(\mathrm{p}<0,001)$. Assim, conclui-se que o método implantado para o controle da esterilização proporcionou maior satisfação e segurança para os alunos.

Descritores: Esterilização. Instituições Acadêmicas. Exposição a Agentes Biológicos. Validação de Programas de Computador.
\end{abstract}




\section{INTRODUÇÃO}

Atualmente, o termo biossegurança ocupa um espaço muito importante na Área da Saúde e consequentemente na Odontologia ${ }^{1}$. Várias são as condutas necessárias para garantir o bloqueio da transmissão de microrganismos patogênicos impedindo a contaminação cruzada ${ }^{2}$. As infecções podem ser transmitidas no consultório odontológico por meio de várias rotas: (1) contato direto com sangue, fluidos orais ou outros materiais infectados, (2) contato indireto com objetos contaminados, como instrumentos, superfícies ambientais ou equipamentos, (3) contato de mucosa conjuntival, nasal ou oral com gotículas, como respingos, contendo microrganismos de uma pessoa infectada e propelidos por tosse, espirro ou fala, ou (4) inalação de microrganismos no ar que podem permanecer suspensos no ar por longos períodos. Ao abordar esses problemas, há duas considerações identificáveis: como o cirurgiãodentista (CD) e sua equipe podem ser salvaguardados da aquisição da doença e da transmissão de doenças aos pacientes e que medidas devem ser tomadas para ajudar a minimizar a contaminação cruzada com instrumentação protética ${ }^{3}$.

Os trabalhos de levantamento de dados de controle de infecção cruzada no país demonstram que tanto acadêmicos de Odontologia ${ }^{4}$ quanto profissionais da área não aplicam adequadamente medidas de biossegurança na prática diária e que os procedimentos de esterilização se mostram falhos e sem protocolo padronizado ${ }^{5,6}$. Assim, o estabelecimento de normas e rotina de biossegurança nos diversos cursos superiores de Odontologia é fundamental, visto que o profissional seguirá as medidas e costumes adotados durante a vida acadêmica ${ }^{1}$. A padronização de procedimentos de controle dos materiais esterilizados e utilização de recursos tecnológicos e de educação continuada são as melhores alternativas para reduzir exposições ocupacionais. Além disso, é necessário prestar uma assistência de qualidade aos pacientes, garantindo a esterilidade de materiais odontológicos e a sua posterior manutenção durante o armazenamento e o manuseio ${ }^{7}$.

Nas universidades, a responsabilidade de fornecer o material esterilizado aos alunos é da Central de Esterilização (CE), à qual cabe submeter o material já lavado e preparado pelos alunos, à esterilização, armazenamento e distribuição. $\mathrm{O}$ controle dos materiais esterilizados e sua distribuição para os alunos representa um grande desafio para as universidades, que têm demonstrado uma preocupação na melhoria destes processos para diminuir os riscos ao operador e aos pacientes $^{8}$, e além disso resolver os problemas internos referentes ao processo como filas da esterilização gerando atrasos para o atendimento dos pacientes. Entretanto, a literatura é escassa sobre os protocolos de esterilização e armazenamento dos materiais adotados pelas universidades.

A Universidade Univille/SC relatou, em 2015 ${ }^{9}$, a implantação de um novo método de controle do processo de esterilização de materiais odontológicos dos alunos. Por meio de um software, os alunos acessam seu login individual, que os permite gerar etiquetas com códigos de barras para identificação dos materiais que são entregues na central de esterilização. Essas etiquetas com todas as informações do aluno e do processo de esterilização são posteriormente fixadas no prontuário do paciente atendido com os respectivos materiais utilizados.

$\mathrm{Na}$ Universidade Positivo, a CE enfrentava dificuldades em relação à forma de recebimento e entrega dos materiais e ao tempo de armazenamento. No antigo sistema, a entrega de materiais ocorria de forma não padronizada, sendo que havia material embalado isoladamente em papel grau cirúrgico, dificultando a colocação na autoclave e favorecendo danos à embalagem. No 
que se refere ao recebimento dos materiais pelos alunos, o método de controle era realizado por meio de canhotos de papel onde constava a matrícula do aluno e o número de pacotes entregues à $\mathrm{CE}$. Em caso de perda do canhoto, para retirar o material era necessário assinar um caderno de controle, deixando o processo de entrega lento e desfavorável, ocasionando filas. Com o objetivo de melhorar o controle do material esterilizado e, além disso, agilizar o processo de entrega e retirada de materiais pelos alunos, foi implantado um novo método sistematizado na central de controle de esterilização.

O objetivo desse trabalho é descrever o novo método de controle da esterilização dos materiais dos alunos de Odontologia e comparar a satisfação dos alunos com o novo método em relação ao antigo.

\section{METODOLOGIA}

\section{Descrição do método de controle de esterilização}

Um software foi desenvolvido pelo serviço de Gestão de Tecnologia da Informação (TI) da Universidade Positivo, denominado sistema de esterilização, com o principal objetivo de controlar a entrada e saída de materiais da CE. Para utilização desse software, foram adquiridos quatro computadores, duas impressoras térmicas de etiquetas Zebra GC420t e quatro leitores de códigos de barras de alta precisão (Honeywell International Inc, modelo MS9520). Esse software permite acesso exclusivo dos funcionários responsáveis pela esterilização e dos professores, com login e senha. Por meio da leitura de códigos de barras, identifica os materiais e os alunos.

Para iniciar a abertura desse protocolo de esterilização, o estudante deve possuir sua carteirinha do aluno com código de barra vinculado ao seu número de matrícula, que já é utilizada por todos os alunos matriculados na Universidade Positivo para o sistema de empréstimo de livros da biblioteca. No momento em que o funcionário lê o código de barras na carteirinha do aluno (figura 1), o sistema gera códigos de barras específicos para aquele aluno. Esses códigos são impressos em etiquetas que serão utilizadas para identificar as caixas de cada aluno permanentemente (figura 2). Estas etiquetas são denominadas BOPP, e apresentam tamanho de 60x40 mm, cor branca e têm como característica suportar calor e vapor durante o processo de esterilização. Assim, é estabelecido que todo o material esterilizado deve estar acondicionado dentro destas caixas identificadas. No código de leitura dessas etiquetas estão vinculadas todas as informações do aluno como nome completo, matrícula ou $\mathrm{CPF}$ e número do escaninho.

Uma segunda etiqueta é impressa com nome, matrícula e escaninho do aluno e o tipo de caixa é identificado de acordo com a especialidade (clínica geral, Dentística, Prótese, Endodontia, Periodontia e Cirurgia). Além disso, para facilitar a identificação e dar agilidade ao trabalho dos funcionários na entrega dos materiais, são inseridas etiquetas de controle com cores (figura 3), identificando o tipo de caixa. Por exemplo, a etiqueta vermelha identifica a caixa de Cirurgia.

Após a correta identificação das caixas, o aluno pode entregar seu material para esterilização, estando devidamente embalado em papel grau cirúrgico. Ao receber o material do aluno, o funcionário fará a leitura da sua carteirinha, dando entrada no sistema (figura 4). Em seguida fará a leitura dos códigos de barra das caixas e o selamento dos pacotes em que estão embalados. Após o selamento, é colocada no pacote uma etiqueta simples de protocolo de esterilização com data, lote e vencimento. No software existe uma aba de controle denominada "autoclave", onde é selecionada a identificação da autoclave na qual este material será esterilizado. Assim, após selecionar o número da autoclave é feito novamente a leitura dos códigos de barra das caixas, e estas são então inseridas para o processo propriamente dito da esterilização. Isso permite que a $\mathrm{CE}$ tenha controle 
sobre todo o processo de esterilização, inclusive da autoclave na qual o material foi inserido. Caso exista qualquer problema em uma autoclave, identificado pelos controles físicos, químicos ou biológicos, é possível gerar relatórios com a identificação dos materiais esterilizados nos últimos ciclos.

Posteriormente ao ciclo de esterilização, o material é encaminhado para a área limpa e colocado nos escaninhos dos alunos. Para retirar seus materiais, o estudante deve apresentar sua carteirinha na CE. O funcionário faz a leitura do código de barra da carteirinha para que o software busque todos os materiais armazenados (figura 5). Nessa página ainda constam informações como nome completo, matrícula, data e hora em que o material foi entregue à $\mathrm{CE}$, data e horário em que foi realizado o ciclo, tipos de caixas que já estão esterilizadas e o número do escaninho do aluno. Além disso, quando o material estiver esterilizado há mais de trinta dias, o sistema acusará o vencimento e o funcionário devolverá o material aberto ao aluno. Após a conferência de todos esses itens, o funcionário faz a leitura dos códigos de todas as caixas entregues para dar baixa no sistema.

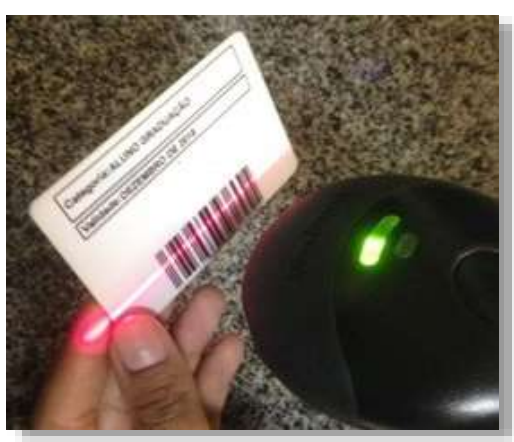

Figura 1. Carteirinha para identificação do aluno

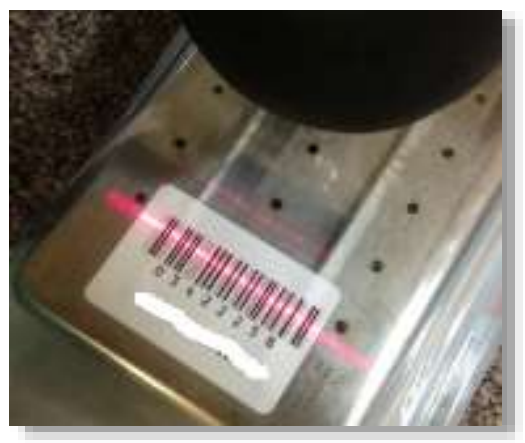

Figura 2. Leitura da etiqueta na central de esterilização

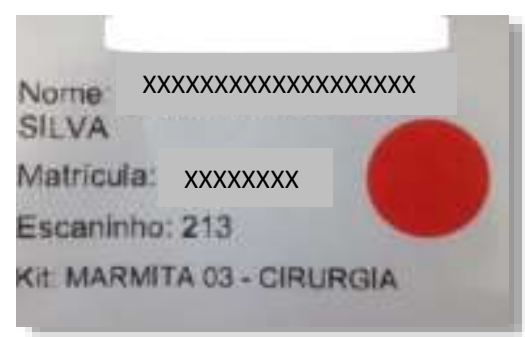

Figura 3. Exemplo de identificação das caixas por cores

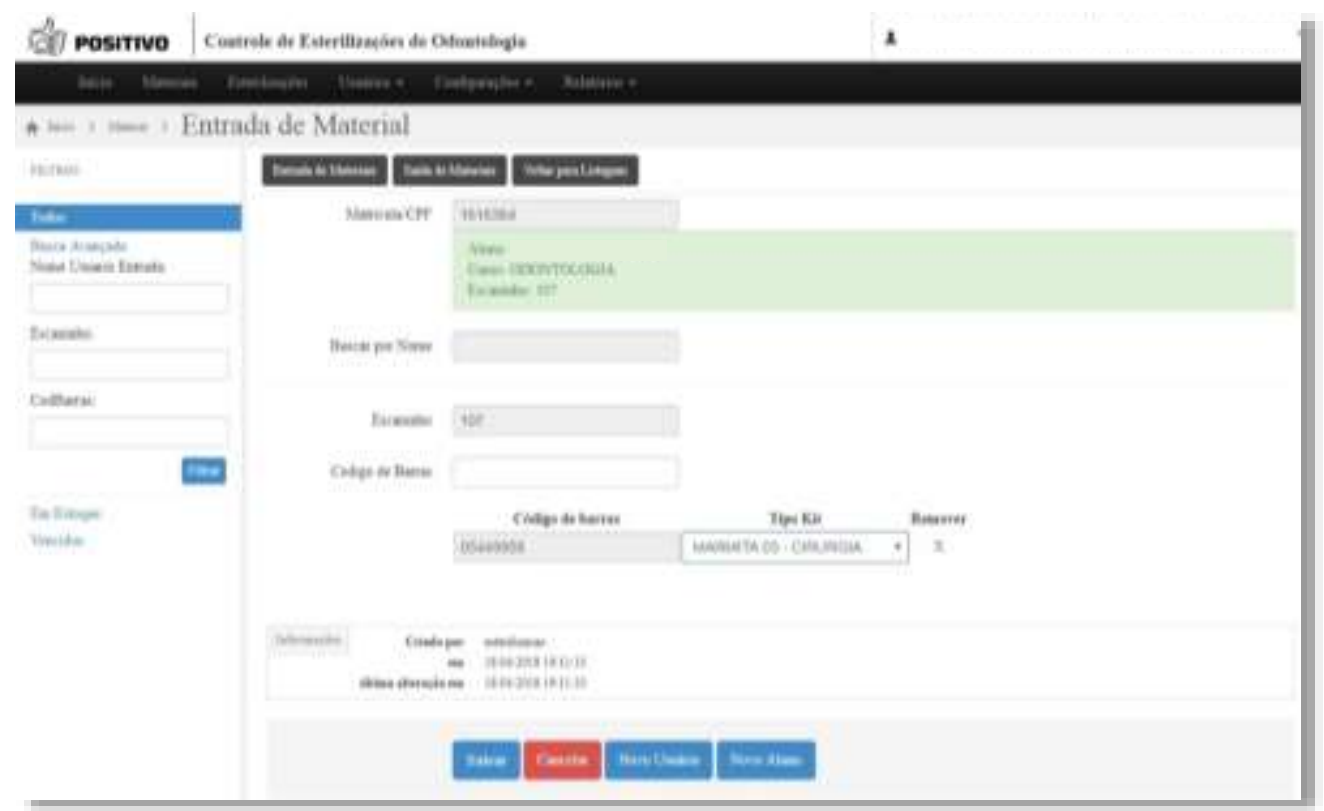

Figura 4. Tela do software exibida após leitura da carteirinha do aluno para a entrega do material 


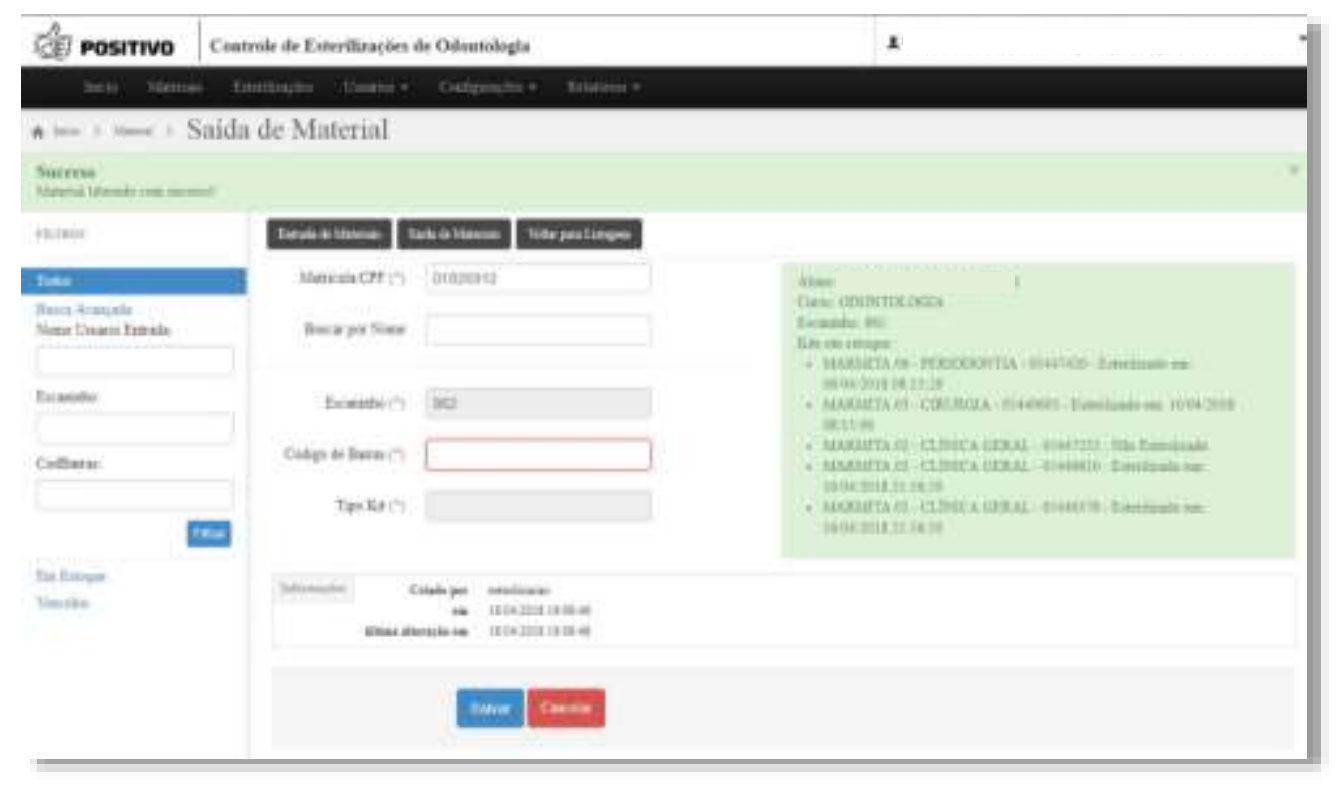

Figura 5. Tela do software exibida após leitura da carteirinha do aluno para a retirada do material

Esse material será entregue e transportado pelo aluno com uma bandeja de plástico até o seu armário limpo, onde será armazenado até o momento do uso. O material deve permanecer somente 30 dias no armário limpo, sendo responsabilidade do aluno averiguar as datas de vencimento.

O software ainda permite outros controles de busca, gerando relatórios sobre as autoclaves, quantidade e tipos de caixas, assim como datas de esterilização de materiais por aluno durante todo o período de matrícula na universidade. Caso um professor deseje verificar se todos os alunos esterilizaram o material para sua disciplina, o sistema gera esse relatório.

\section{Avaliação do software pelos alunos}

A pesquisa foi aprovada pelo Comitê de Ética em Pesquisa da Universidade Positivo sob o protocolo CAAE: 80721117.5.0000.0093.

Para avaliarmos a aceitação e a qualidade do novo método implantado, foi aplicado um questionário aos alunos de graduação do último ano de Odontologia, pois estes alunos utilizaram ambos os métodos de controle (antigo e o atual/software), possibilitando a comparação entre eles. Todos os alunos que aceitaram participar da pesquisa e assinaram o termo de consentimento livre e esclarecido foram incluídos.

Em relação aos sistemas de esterilização, os alunos foram questionados sobre atrasos no atendimento aos pacientes decorrentes de filas para a retirada de materiais na CE. Também foram questionados sobre perdas ou danificações de materiais e sobre se sentirem seguros em deixar seus materiais na esterilização. Para estas perguntas, as possibilidades de respostas eram sim e não. Também foi questionado o tempo médio gasto pelos alunos na colocação e retirada de materiais na $\mathrm{CE}$, apresentando como opções de resposta dez, quinze, trinta e quarenta minutos. Foi solicitado ainda que os alunos estabelecessem uma nota de um a dez para ambos os sistemas, além de classificá-los como ótimo, bom, regular e péssimo.

Os resultados obtidos foram submetidos à análise descritiva e estatística. Para comparação entre os sistemas com relação às variáveis 
dicotômicas, obtidas pelas perguntas com respostas sim e não, foi utilizado o teste do Quiquadrado. Para avaliar a normalidade de distribuição das variáveis numéricas foi utilizado o teste Kolmogorov-Smirnov. Para comparação das medianas de tempo de espera para colocação e retirada entres os sistemas e da nota dada a cada sistema, foi utilizado o teste Mann-Whitney. Para comparação das variáveis ordinais entre os grupos, também foi utilizado o teste MannWhitney. Valores de $p<0,05$ indicaram significância estatística. Os dados foram analisados com o programa computacional IBM
SPSS Statistics v.24.

\section{RESULTADOS}

A tabela 1 demonstra o percentual de alunos que relataram a ocorrência de atrasos para $o$ atendimento de pacientes e perda ou danos de materiais, no antigo e novo método de controle. A maioria deles relatou atrasos com o método antigo. Já com relação ao método novo o relato de atrasos diminuiu significativamente. A ocorrência de materiais perdidos ou quebrados também foi menor no sistema novo comparado ao sistema antigo.

Tabela 1. Prevalência de atrasos e problemas com materiais com os métodos antigo e novo de controle de esterilização

\begin{tabular}{r|ccc}
\hline & $\begin{array}{c}\text { Método antigo } \\
\mathbf{n}(\boldsymbol{\%})\end{array}$ & $\begin{array}{c}\text { Método novo } \\
\mathbf{n}(\boldsymbol{\%})\end{array}$ & $\begin{array}{c}\text { Valor de } \\
\mathbf{p}^{*}\end{array}$ \\
\hline Atrasos em atendimentos & $95(85,6)$ & $32(28,8)$ & $<0,001$ \\
Materiais quebrados ou perdidos & $54(49,1)$ & $16(14,5)$ & $<0,001$ \\
\hline
\end{tabular}

*Teste do Qui-quadrado.

Em relação à pergunta sobre sentirem-se seguros em deixar os materiais na esterilização, verifica-se que $85,6 \%$ dos alunos demonstraram sentir segurança com o novo sistema, enquanto apenas $34,2 \%$ relataram que sentiam segurança no antigo sistema $(p<0,001)$.

No que se refere ao tempo de fila para colocação do material pelos alunos, notou-se que para o antigo método foi encontrado uma mediana de 15 (5-40), enquanto que para o novo método a mediana foi menor $(p<0,001)$, totalizando 5 (5-30) minutos. Quanto ao tempo para retirada do material, também foi relatado com uma mediana de 15(5-40) minutos no antigo método, e 5 (5-30) minutos no novo método de esterilização, demonstrando novamente maior agilidade no novo método $(p<0,001)$.

Com relação à nota dada para cada sistema pelos alunos, que podia variar de 0 a 10, para o antigo método a mediana foi 5 (1-10), enquanto para o novo método a mediana foi maior com um valor de 9 (5-10), $(p<0,001)$.

O gráfico 1 demonstra a distribuição dos dois sistemas de acordo com classificação de qualidade dada pelos alunos. Para o método antigo a maioria das respostas corresponderam à regular e para o novo método, a maioria das respostas para o novo método foram bom e ótimo $(p<0,001)$.

\section{DISCUSSÃO}

Os cursos de Odontologia atualmente se deparam com vários desafios no controle de esterilização de materiais para o atendimento clínico pelos estudantes. Dificuldades no controle de entrada e saída de materiais, controle da esterilização do material utilizado e o 
armazenamento dos materiais são alguns dos problemas encontrados.

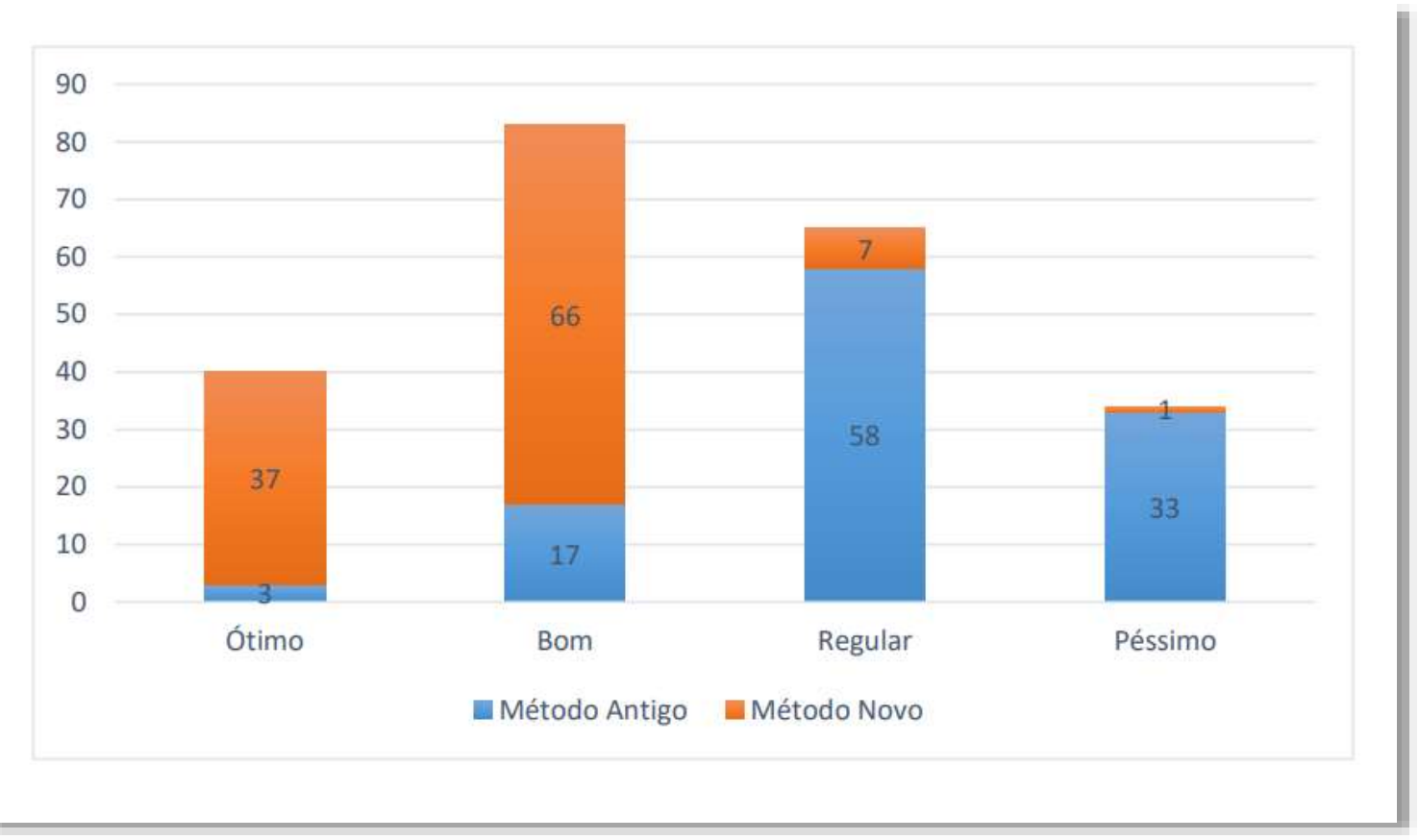

Gráfico 1. Distribuição das frequências de respostas dos alunos em relação aos métodos de controle de esterilização (teste de Mann-Whitney, p<0,001).

O controle de entrada e saída dos materiais demonstrou ser mais ágil e efetivo com um método sistematizado. Outras vantagens do novo método são somente permitir a entrega e retirada pelo aluno que possui as respectivas caixas registradas, garantindo maior organização para a entrega, evitando extravio de material e diminuindo custos de reposição de materiais. Por meio dos questionários aplicados aos alunos foi possível verificar que eles se sentem mais seguros com o novo método, relatando menor índice de perdas, danos e atrasos.

À respeito do controle de esterilização, de acordo com Guandalini e colaboradores ${ }^{10}$, em 1999, o monitoramento biológico é capaz de assegurar a efetividade da esterilização, devendo ser feito semanalmente, sendo seu resultado registrado e mantido em arquivos de controle. Diversos estudos têm sido realizados para testar a efetividade das autoclaves ${ }^{11}$. Entretanto, apenas o correto ciclo de esterilização dos materiais não garante a segurança do material estéril até o atendimento clínico do paciente. $\mathrm{O}$ acondicionamento e armazenamento correto dos materiais, a entrada e saída dentro dos prazos de conservação também deve receber um rigoroso monitoramento pela CE. A implantação de um método sistematizado eletronicamente, facilita o controle destes registros para cada aluno individualmente.

O método relatado pela universidade Univille/SC ${ }^{9}$ demonstra ter um maior controle dos materiais a partir da saída da CE até o atendimento do paciente, já que todos os materiais retirados recebem etiquetas constando a data de esterilização e o prazo de validade, que são coladas no prontuário do paciente. Esse método foi a base para construção do novo 
sistema da Universidade Positivo, sendo a principal diferença entre eles alguns atalhos que o software criado pela Universidade Positivo oferece, os quais garantem melhor acompanhamento por parte dos professores do material que foi esterilizado durante toda a permanência do estudante no curso. O software ainda controla os materiais que estão com a esterilização vencida.

Com relação à forma de organização dos materiais que devem ser esterilizados, Pimentel e colaboradores $^{12}$ relataram em 2012 que os estudantes não têm o hábito de separar seus instrumentais por procedimentos ou por número de pacientes previamente programados. Relataram ainda que $31,6 \%$ deles esterilizam todo o material em uma caixa única e usam o instrumental não utilizado no primeiro atendimento em outro paciente. Sabe-se que mesmo materiais não utilizados no paciente são considerados contaminados após a abertura do pacote pela deposição de aerossóis oriundos de procedimentos clínicos. A segurança do paciente depende de instrumentos adequadamente preparados $\mathrm{e}$ esterilizados $^{13}$. A nova metodologia implantada na Universidade Positivo, controla a separação das marmitas de acordo com cada especialidade. Além de ajudar na organização dos alunos, isso diminui a probabilidade de o aluno utilizar instrumentais em outros pacientes, após a marmita já ter sido aberta. Sabe-se que a melhor garantia de um produto estéril é uma execução cuidadosa de cada etapa do processo, juntamente com um programa de controle de qualidade contínuo ${ }^{14}$.

Além de o novo método demonstrar maior efetividade para controlar que todos os materiais utilizados pelos alunos estejam estéreis, os alunos demonstraram de uma forma geral uma satisfação maior com o novo método implantado.

\section{CONCLUSÃO}

O novo método parece facilitar o controle de funcionários e professores sobre a esterilização dos materiais utilizados pelos alunos. Além disso, resultou em maior satisfação dos estudantes em relação à menor ocorrência de atrasos, de perdas e danos de materiais, proporcionando maior segurança para deixar os materiais para serem esterilizados e menor tempo para sua entrega e retirada.

\section{ABSTRACT \\ Implantation of software to control the sterilization department of the Dental School of Positivo University}

This study aims to describe a method for controlling the sterilization of materials used by dentistry students and to compare its efficiency against the former control method. A software was developed by the Information Technology Management Department at the Positivo University, motivated by an internal demand to improve the sterilizing department control. The new method allows the placement of labels with bar codes on the external surface of the students' boxes, controls the entire entrance-and-exit flow of materials and provides greater safety and agility for the sterilization process. Furthermore, the system allows professors to access the list of materials sterilized by students, and the date, time, and number of the autoclave in which the material was sterilized. Questionnaires including subjective questions about the new and former method of control were administered to final-year dentistry students to evaluate their satisfaction toward the methods. The new method decreases delays in patient care and the number of damaged and lost materials, increases safety, and reduces the time spent in material drop-off and withdrawal ( $\mathrm{p}$ $<\square 0.05$ ). Generally, the new method is considered better when compared to the former one ( $p$ $<\square 0.001$ ). Therefore, we can conclude that the method implemented to control sterilization resulted in greater student satisfaction and provided greater safety.

Descriptors: Sterilization. Schools. Exposure to Biological Agents. Software Validation.

\section{REFERÊNCIAS}

1. Su J, Deng XH, Sun Z. A 10-year survey of compliance with recommended procedures 
for infection control by dentists in Beijing. Int Dent J. 2012; 62(3):148-53.

2. Pimentel MJ, Filho MMVB, Santos JP, Rosa MRD. Biossegurança: comportamento dos alunos de Odontologia em relação ao controle de infecção cruzada. Cad Saúde Colet. 2012; 20(4):525-32.

3. Upendran A, Bhimji SS. Dental, Infection Control. Treasure Island (FL): StatPearls Publishing 2017, 29.

4. Lima AA, Azevedo AC, Fonseca AGL, Silva JLM, Padilha WWN. Acidentes Ocupacionais Entre Estudantes de Odontologia. Pesq Bras Odontoped Clin Integr. 2008; 8(3):327-32.

5. Naressi SCM, Akama CM, Silva LMP, Siviero M. Análise das formas de esterilização e do meio de controle empregados pelos cirurgiões-dentistas de São José dos Campos - SP. Rev Odontol UNESP. 2004; 33(4):169-74.

6. Prado MEM, Santos SSF. Avaliação das condições de esterilização de materiais odontológicos em consultórios na cidade de Taubaté. Rev Biociênc. 2002;8(1):61-70.

7. Oliveira CAS, Costa SM, Zocratto KBF, Branco KMGR. Avaliação microbiana da recontaminação de artigos odontológicos estéreis segundo o manuseio das embalagens. RFO UPF. 2011;16(3):256-60.

8. Demenech LS, Tomazinho FSF, Tomazinho PH, Gabardo MCL, Baratto-Filho F. Avaliação de métodos de manutenção da esterilidade do órgão dental humano extraído para armazenamento em banco de dentes. Rev ABENO. 2017;17(3):55-65.
9. Univille introduz uso de etiquetas para os prontuários de pacientes. ABENO news 2015, 47. [Acesso em 8 jul. 2018]. Disponível em: http://www.abeno.org.br/abeno-ews/abenonews47.html.

10. Guandalini SL, Melo NSFO, Santos ECP. Biossegurança em Odontologia. 2ed. Curitiba: Odontex, 1999, 161p.

11. O'Hara NN, Patel KR, Caldwell A, Shone S, Bryce EA. Sterile reprocessing of surgical instruments in low- and middle-income countries: A multicenter pilot study. Am J Infect Control. 2015; 43(11):1197-200.

12. Pimentel M J, Batista Filho MMV, Santos JP, Rosa MRD. Biossegurança: comportamento dos alunos de Odontologia em relação ao controle de infecção cruzada. Cad Saúde Colet. 2012; 20(4):525-32.

13. Seavey R. High-level disinfection, sterilization, and antisepsis: current issues in reprocessing medical and surgical instruments. Am J Infect Control. 2013;41(5 Suppl):S111-7.

14. Spry C. Understanding current steam sterilization recommendations and guidelines. AORN J. 2008;88(4):537-50; quiz 551-4.

Correspondência para:

Rafaela Scariot

E-mail: moraes@up.edu.br

Rua Prof. Pedro Viriato Parigot de Souza, 5300

Cidade Industrial

81280-330 Curitiba/PR 